\title{
ReaR
}

\section{Bloqueo TAP medioaxilar como tratamiento analgésico en el proceso de obtención de injerto de cresta ilíaca.}

Benítez Jiménez M, Alcaraz Martínez AB, García Sánchez F, Granskog Sierra A.

Hospital General Universitario José María Morales Meseguer, Murcia.

\begin{abstract}
Resumen
Entre los problemas más frecuentes tras la obtención del injerto de cresta (ICI) se encuentran la alta incidencia de dolor postoperatorio agudo y la mayor prevalencia de dolor crónico en el área de la incisión que, según algunas series de casos, puede alcanzar hasta el 40\%. El bloqueo TAP medioaxilar, el cual actúa sobre las raíces nerviosas de T7 a L1, puede ser eficaz para controlar el dolor de la incisión y de la manipulación de la cresta ilíaca.

En este trabajo, presentamos tres casos de artroplastia de hombro que requirieron ICI. En todos ellos se utilizó la combinación de dos técnicas de anestesia locorregional, el bloqueo TAP media axilar y el bloqueo del plexo braquial a nivel interescalénico, con el objetivo de evitar la aparición de dolor intra y postoperatorio, evitando el uso de opioides. Además, en ningún caso hubo signos de intoxicación sistémica por anestésicos locales.
\end{abstract}

\section{Introducción}

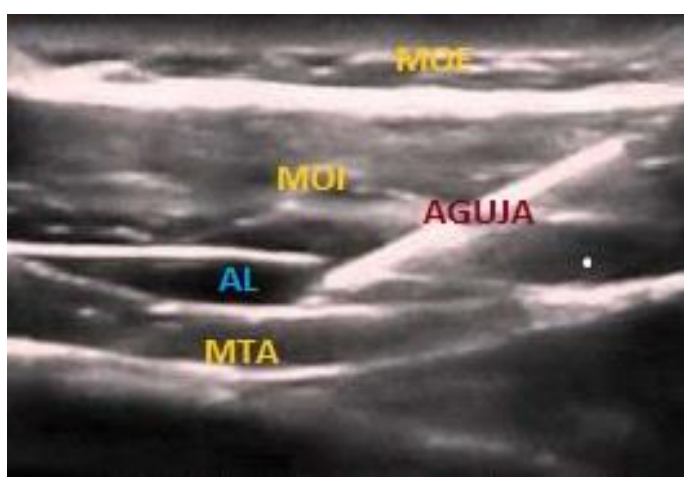

Entre los problemas más frecuentes tras la obtención del injerto de cresta (ICI) se encuentran la alta incidencia de dolor postoperatorio agudo y la mayor prevalencia de dolor crónico en el área de la incisión que, según algunas series de casos, puede alcanzar hasta el $40 \%$. El bloqueo TAP medioaxilar, el cual actúa sobre las raíces nerviosas de $\mathrm{T} 7 \mathrm{a}$ L1, puede ser eficaz para controlar el dolor de la incisión y de la manipulación de la cresta ilíaca.
En este trabajo, presentamos tres casos de artroplastia de hombro que requirieron ICI. En todos ellos se utilizó la combinación de dos técnicas de anestesia locorregional, el bloqueo TAP media axilar y el bloqueo del plexo braquial a nivel interescalénico, con el objetivo de evitar la aparición de dolor intra y postoperatorio, evitando el uso de opioides. Además, en ningún caso hubo signos de intoxicación sistémica por anestésicos locales.

En determinadas artroplastias se pueden requerir injertos óseos para lograr la estabilidad total de la articulación, siendo el injerto óseo más utilizado el de cresta ilíaca (CI) autólogo. La artroplastia de hombro es una de las cirugías que más se beneficia de este procedimiento.

Uno de los problemas más frecuentes tras la obtención del injerto de cresta ilíaca (ICI) es el dolor agudo postoperatorio (1), que puede aparecer hasta en el 39\% de los pacientes (2), 
según las series. Otro problema bastante frecuente es el de la alteración sensitiva en la zona de obtención del injerto, pudiendo desencadenar dolor crónico hasta en el $40 \%$ de los casos (3). La incisión realizada para la obtención del ICI lesionará la piel y músculos de la pared abdominal en relación con la $\mathrm{CI}$, además de estructuras nerviosas tales como los nervios iliohipogástrico e ilioinguinal (originado en la rama anterior de L1), cuyo recorrido se encuentra próximo a la CI. Es por ello que el bloqueo TAP medioaxilar, el cual actúa sobre las raíces nerviosas desde T7 a L1 (Fig. 1), resultaría efectivo en la prevención de aparición del dolor tras la incisión realizada y manipulación de la CI, evitando así los posibles fenómenos de sensibilización que darían lugar a la posterior aparición de dolor crónico.

En este trabajo presentamos tres casos de artroplastia de hombro que requirieron ICI. En todos se empleó la combinación de dos técnicas de ALR, con el objetivo de evitar la aparición de dolor intra y postoperatorio, evitando el uso de opioides. Por un lado, se realizó un bloqueo TAP mediaxilar ecoguiado (Fig.2) para manejo del dolor secundario a la obtención del ICI; y por otro, se asoció un bloqueo del plexo braquial a nivel interescalénico (Fig.3) como técnica analgésica para la cirugía del hombro.

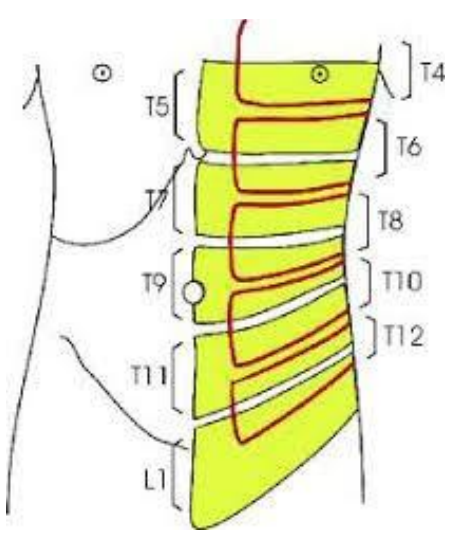

Figura1.

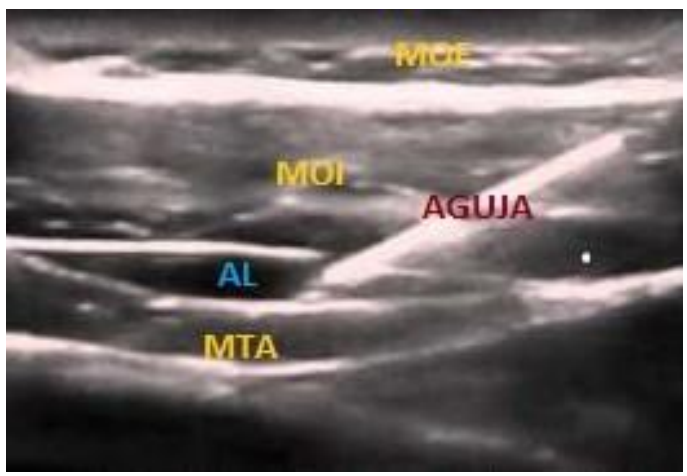

Fig. 2. MOE: músculo oblicuo externo. MOI: músculo oblicuo interno. MTA: músculo transverso del abdomen. AL: anestésico local.

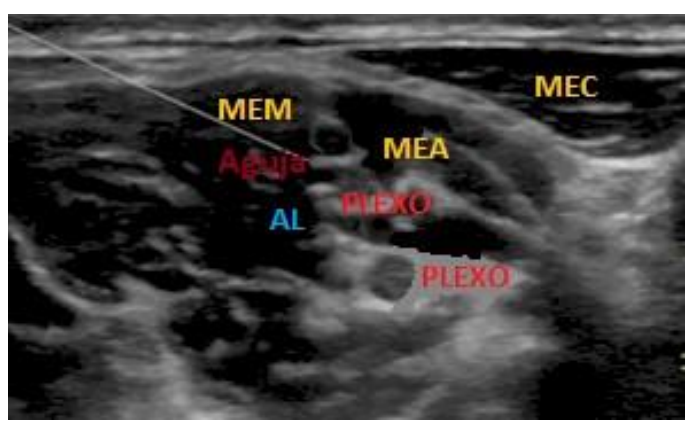

Fig. 3. MEC: músculo esternocleidomastoideo. MEM: músculo escaleno medio. MEA. Músculo escaleno anterior. AL: anestésico local.

\section{Caso clínico}

En los tres casos se realizó una cirugía de hombro coninjerto autólogo de CI para lograr la total estabilización de la articulación. Los datos de los pacientes se recogen en la tabla 1 :

\begin{tabular}{|c|c|c|c|}
\hline & Paciente 1 & Paciente 2 & Paciente 3 \\
\hline Edad & 70 & 75 & 72 \\
\hline Peso & 87 & 93 & 82 \\
\hline AP de interés & $\begin{array}{l}\text { EPOC } \\
\text { moderado- } \\
\text { grave - SAHS } \\
\text { sin CPAP }\end{array}$ & $\begin{array}{l}\text { SAHS con } \\
\text { CPAP } \\
\text { nocturna }\end{array}$ & $\begin{array}{l}\text { EPOC } \\
\text { moderado- } \\
\text { grave }\end{array}$ \\
\hline $\begin{array}{l}\text { Predictores } \\
\text { de VAD }\end{array}$ & $\begin{array}{l}\text { Flexión- } \\
\text { extensión } \\
\text { cervical } \\
\text { limitada }\end{array}$ & $\begin{array}{l}\text { Mallampati } \\
\text { III Distancia } \\
\text { TM }<6 \mathrm{~cm}\end{array}$ & $\begin{array}{l}\text { Flexión- } \\
\text { extensión } \\
\text { cervical } \\
\text { limitada }\end{array}$ \\
\hline
\end{tabular}

Tabla 1. AP: antecedentes personales. VAD: vía aérea difícil. EPOC: enfermedad pulmonar obstructiva crónica. SAHS: síndrome de apnea hipoventilación. CPAP: presión positiva continua en la vía aérea. TM: tiromentoniana.

Se premedicó a los pacientes con omeprazol $40 \mathrm{mg}$ iv, dexametasona 8 
$\mathrm{mg}$ iv, metoclopramida $10 \mathrm{mg}$ iv $\mathrm{y}$ lorazepam $1 \mathrm{mg}$ vo.

De cara a la intervención sobre el hombro, se optó por la realización de un bloqueo del plexo braquial a nivel interescalénico ecoguiado previo a la inducción anestésica, para el que se administraron $20 \mathrm{ml}$ de la mezcla anestésica con mepivacaína $1 \%(10 \mathrm{ml})$ y levobupivacaina $0.375 \%(10 \mathrm{ml})$, con aspiración y dosis fraccionada, que resultó efectivo en los tres casos. Tras esto, se procedió a la anestesia general, realizando una inducción inhalatoria con sevoflorano, con el objetivo de mantener la ventilación espontánea. Para el manejo de la vía aérea se empleó mascarilla laríngea tras alcanzar el plano IV de Guedel. En ningún caso fue necesario el uso de relajantes neuromusculares. El mantenimiento anestésico también se hizo con sevoflorano, asociado a la perfusión continua de remifentanilo.

Tras la inducción anestésica se realizó el bloqueo TAP medioaxilar ecoguiado, para prevenir la aparición de dolor causada por la manipulación sobre la CI. En el bloqueo TAP se volvió a emplear $20 \mathrm{ml}$ de la mezcla anestésica previamente descrita. De esta manera, la dosis total de AL administrada fue de $400 \mathrm{mg}$ de mepivacaína y $150 \mathrm{mg}$ de levobupivacaína. Teniendo en cuenta el peso de los pacientes y las dosis máximas para los $\mathrm{AL}$ administrados (tabla 2), en ningún caso se superó la dosis máxima recomendada (tabla 3 ).

\section{AL Levobupivacaína Mepivacaína \\ Dosis máxima $2,5 \mathrm{mg} / \mathrm{kg}^{*} \quad 5 \mathrm{mg} / \mathrm{kg} *$}

Tabla 2. Dosis máxima de AL por kilogramo de peso.

*En función de la bibliografía consultada las dosis máximas pueden variar. En el caso de la mepivacaína se recomienda no superar los $400 \mathrm{mg}$ en total, independientemente del peso del paciente. Para la levobupivacaína se recomienda no superar los $150 \mathrm{mg}$ en dosis única, independientemente del peso. AL: anestésico local.

\begin{tabular}{|c|c|c|}
\hline $\begin{array}{l}\text { Peso } \\
(\mathrm{kg})\end{array}$ & $\begin{array}{l}\text { Dosis máxima } \\
\text { levobupivacana }\end{array}$ & $\begin{array}{l}\text { de Dosis máxima de } \\
\text { mepivacaína }\end{array}$ \\
\hline 87 & $217,5 \mathrm{mg}$ & $435 \mathrm{mg}$ \\
\hline 93 & $232,5 \mathrm{mg}$ & $465 \mathrm{mg}$ \\
\hline 82 & $205 \mathrm{mg}$ & $410 \mathrm{mg}$ \\
\hline
\end{tabular}

Tabla 3. Dosis máxima de AL para cada uno de nuestros pacientes en función del peso.

Media hora antes de la finalización de la intervención, se administró paracetamol $1 \mathrm{gr}$, tramadol $1 \mathrm{mg} / \mathrm{kg}$ y metamizol 2 gr iv y profilaxis de NVPO con ondansetron $4 \mathrm{mgr}$. Tras la educción anestésica los pacientes fueron trasladados a la URPA donde se registró el EVA a la primera hora y a su salida, siendo ambos de 0; tanto en el hombro como en el área de la cresta ilíaca. A las 24 horas continuaban con EVA 0 con pauta analgésica: paracetamol iv $1 \mathrm{gr}$ iv y metamizol 2 gr iv intercalados ambos cada 8 horas, sin precisar analgesia de rescate en los tres casos. Por otro lado, también observamos que a pesar de la dosis de AL empleada no se registró, en ninguno caso, signos de toxicidad sistémica asociada a los anestésicos locales.

\section{Discusión}

Una de las consecuencias de la obtención ICI es el dolor agudo postoperatorio severo, valorado por la mayoría de nuestros pacientes como EVA $>7$. Además un mal manejo del dolor agudo podría dar lugar a la cronificación del mismo por fenómenos de sensibilización central o periférica (4)(5), afectando en algunas series de casos a gran número de pacientes.

La inervación de la zona donde se realiza la incisión para obtener el ICI 
corre a cargo de nervios espinales desde T10 hasta L1, es por ello que un correcto bloqueo TAP medioaxilar resultaría efectivo como tratamiento analgésico en este procedimiento, evitando al mismo tiempo fenómenos de cronificación de dolor por lesión directa de nervios intercostales, así como de los nervios ileohipogástrico e ilioinguinal cuyo recorrido anatómico discurre por esta región de la pared abdominal.

Gracias al empleo de una anestesia multimodal, combinando técnicas locorregionales y analgesia iv con AINEs, es posible la reducción del empleo de opioides, disminuyendo por tanto la incidencia de efectos secundarios degradables derivados de estos (prurito, náuseas, vómitos, sedación...) que dilatan la recuperación anestésica de nuestros pacientes. En nuestro caso el empleo de ambos bloqueos logró un adecuado control del dolor tanto intraoperatorio (no se superó los $0.05 \mathrm{mcrg} / \mathrm{k} / \mathrm{min}$ de remifentanilo) como del posoperatorio, transcurriendo este último sin necesidad de rescate con mórficos.

Es de gran importancia reseñar que, a pesar de combinar dos técnicas de ALR en las que se empleó un volumen importante de AL (40 $\mathrm{ml}$ en total) con mezcla de dos AL diferentes (mepivacaína y levobupivacaína), no se alcanzaron dosis tóxicas de ninguno de ellos. La dosis máxima de mepivacaína se establece en $5 \mathrm{mg} / \mathrm{kg}$ de peso y la de levobupivacaína en $3 \mathrm{mg} / \mathrm{kg}$ de peso, estas dosis ascienden a $7 \mathrm{mg} / \mathrm{kg}$ y 4 $\mathrm{mg} / \mathrm{kg}$ respectivamente si el AL se administra junto a un vasoconstrictor (6), aunque estos datos pueden variar según las fuentes. El uso de una dosis correcta, junto al empleo de otras técnicas de prevención (uso de ultrasonidos, aspiración y dosis fraccionada) evitó la aparición de toxicidad sistémica asociada al uso de
AL(7), de manera que no se registraron signos de neurotoxicidad o cardiotoxicidad en ningún momento.

\section{Conclusión}

El empleo del bloqueo TAP medioaxilar ecoguiado podría resultar beneficioso para prevenir la aparición de dolor intra y postoperatorio, en el proceso de obtención de ICI, pudiendo evitar el uso de opioides y los efectos secundarios derivados de estos.

En nuestros pacientes, la combinación de dos técnicas de ALR; bloqueo TAP y bloqueo del plexo braquial a nivel interescalénico ecoguiadas, empleando un volumen total de $40 \mathrm{ml}$ de AL, 400 mg de mepivacaína y $150 \mathrm{mg}$ de levobupivacaína resultaron seguras, sin evidenciarse signos de intoxicación sistémica por AL.

\section{Bibliografía}

1. Dimitriou R, Mataliotakis GI, Angoules AG, Kanakaris NK, Giannoudis PV. Complications following autologous bone graft harvesting from the iliac crest and using the RIA: a systematic review. Injury. 2011; 42 (2):3-15. ( PubMed)

2. Evan D, Sheha MD, Dennis S, Meredith MD, Grant D. et al. Postoperative pain following posterior iliac crest bone graft harvesting in spine surgery: a prospective, randomized trial. Spine Journal, 2018; 18 (6): 986-992. (HTML)

3. Dennis MD, Bible JE, Crosby CG, Devin JC. Bupivacaine for Pain Reduction After Iliac Crest Bone Graft Harvest. Orthopedics. 2014; 37(5):428434.

4. Kato F, Sugimura YK, Takahashi Y. Pain-Associated Neural Plasticity in the Parabrachial to Central Amygdala Circuit : Pain Changes the Brain, and the Brain Changes the Pain. Adv Exp Med Biol. 2018;1099:157-166. (PubMed)

5. Lavand'homme P, Estebe JP .Opioidfree anesthesia: a different regard to anesthesia practice. Curr Opin Anaesthesiol. 2018 Oct;31(5):556-561. (PubMed) 
6. http://arydol.com/temas/neuroestimula cion/generalidades/anestesicoslocales/.

7. ASRA Checklist Improves Trainee Performance During a Simulated Episode of Local Anesthetic Systemic Toxicity. Neal M, Hsiung L, Mulroyl F, Halpern B, Dragnich D, Slee E. Regional Anesthesia \& Pain Medicine. 37(1):8-15 2012. (PubMed)
Correspondencia al autor

Mercedes Benitez Jiménez

merche 2112@,hotmail.com

MIR Anestesia, Reanimación y Terapeútica del Dolor.

Hospital General Universitario José María Morales Meseguer, Murcia.

Fernando García Sánchez

fernando_garcia72@hotmail.com

FEA Anestesia, Reanimación y Terapeútica del Dolor.

Hospital General Universitario José María Morales Meseguer, Murcia.

Aceptado para el blog en febrero de 2020 